\title{
Video Surveillance Based Attendance system
}

\author{
Mr. Rahul V. Patil ${ }^{1}$, Mrs. S. B. Bangar ${ }^{2}$ \\ PG Research Student, Department of Computer Science \& Technology, Dr. BAMU, Maharashtra Institute of \\ Technology, Aurangabad (MS), India ${ }^{1}$ \\ Assistant Professor, Department of Computer Science \& Technology, Dr. BAMU, Maharashtra Institute of \\ Technology, Aurangabad (MS), India ${ }^{2}$
}

\begin{abstract}
Attendance management system it is very important and popular method to maintain the record of work hours of everyone in organization. The attendance management system is used for multiple areas such as college, university, business organizations. It is very simple method to calculate the attendance how many people are present or absent. Schools and colleges are using attendance system so that they will give marks to students based on their attendance at submission time. Multiple methods are available for calculating attendance i.e. Attendance using pen and paper, fingerprint attendance system, RFID based attendance system, wireless iris recognition attendance management system, attendance management system using SMART-FR, automated attendance management system using face recognition, real time human face detection and tracking, robust and real time face detection.
\end{abstract}

Keywords: RFID, IRIS, SMART-FR.

\section{I . INTRODUCTION}

The success of automated attendance management system using surveillance camera mainly depends on how efficiently, we implement face detection and face recognition algorithm. There are multiple algorithms are available for face detection and recognition system, but these algorithms have some limitations. So the main challenge is overcome through the limitations of existing face detection and face recognition algorithms.

Face recognition is a big research area which takes more attention of many researchers in computer technology. The human face recognition from video sequences it is a challenging task because there are variation present in the background images, facial expression and illumination. Most of the face detection techniques focus on detecting the frontal face of humanity and ignore other things like buildings, Trees and background images.

Generally, the face recognition system is used for the security purpose, but now a days this system is used in multiple other applications. The main purpose of the automated attendance system has recorded the present of peoples inside the organization. The automated attendance system is used to reduce the manual attendance work and time used for maintaining attendance records. In this system, the high resolution surveillance camera is used for capture the video in a classroom. By using this video the attendance of students is pointed. And also, this system is generating the attendance reports as needs of academics.

The rest of the paper is organized as follows: section II covers the related work. The methodology is described in section III. Section IV shows the Experimental results. Finally, concluding remarks are presented in section V.

\section{RELATED WORK}

Several ways are used before to take attendance of students in a classroom. The traditional way is to take attendance by teacher manually by using pen and paper. But limitation with this approach is that its protracted process and there may be the loss of attendance records in future.

Another approach is a fingerprint based attendance system in which a portable device capable of taking students fingerprint is moved inside the classroom. The advantage of this approach is that attendance we will take at lecture time without the instructor's intervention and this system guarantees the marking of full proof attendance. But the problem with the approach is that if we pass the device during lecture time, then there may be distracting of attention of both teacher and student and student interaction required to have attended.

First approach to take the attendance is RFID (Radio Frequency Identification) based attendance management system [4] in which each student will have one unique identity card. That card will be swapped in a machine to put attendance. Swap machine is directly connected to a system that stores attendance related details. The limitation of the above approach is that unauthorized person also can put the attendance.

Second approach is iris based attendance management system [5].The limitation are fingerprint based attendance system is that the fingerprints of children are difficult to scan and time progresses, there may be variation in the fingerprints and many people may have improper fingerprints that may not be suitable for fingerprint based attendance system. So the solution to above limitation is 
iris based attendance system because the eye is most protected part of the body and does not go through much over a complete life of a human being. However, face recognition can be done automatically from video of classroom. Face Recognition System hasn't required active participation from students to put the attendance. With the help of the camera when we capture a face that face will be stored in a device with minimum dimensions, hence the space required for storage is also less.

\section{METHODOLOGY}

The automated attendance management system helps to put attendance of the student when the face is recognized using video surveillance. The existing attendance system has several limitations, so we propose the attendance system in such a way that it will remove the limitations of the existing system. The flow chart of proposed system in shown in Fig 1.

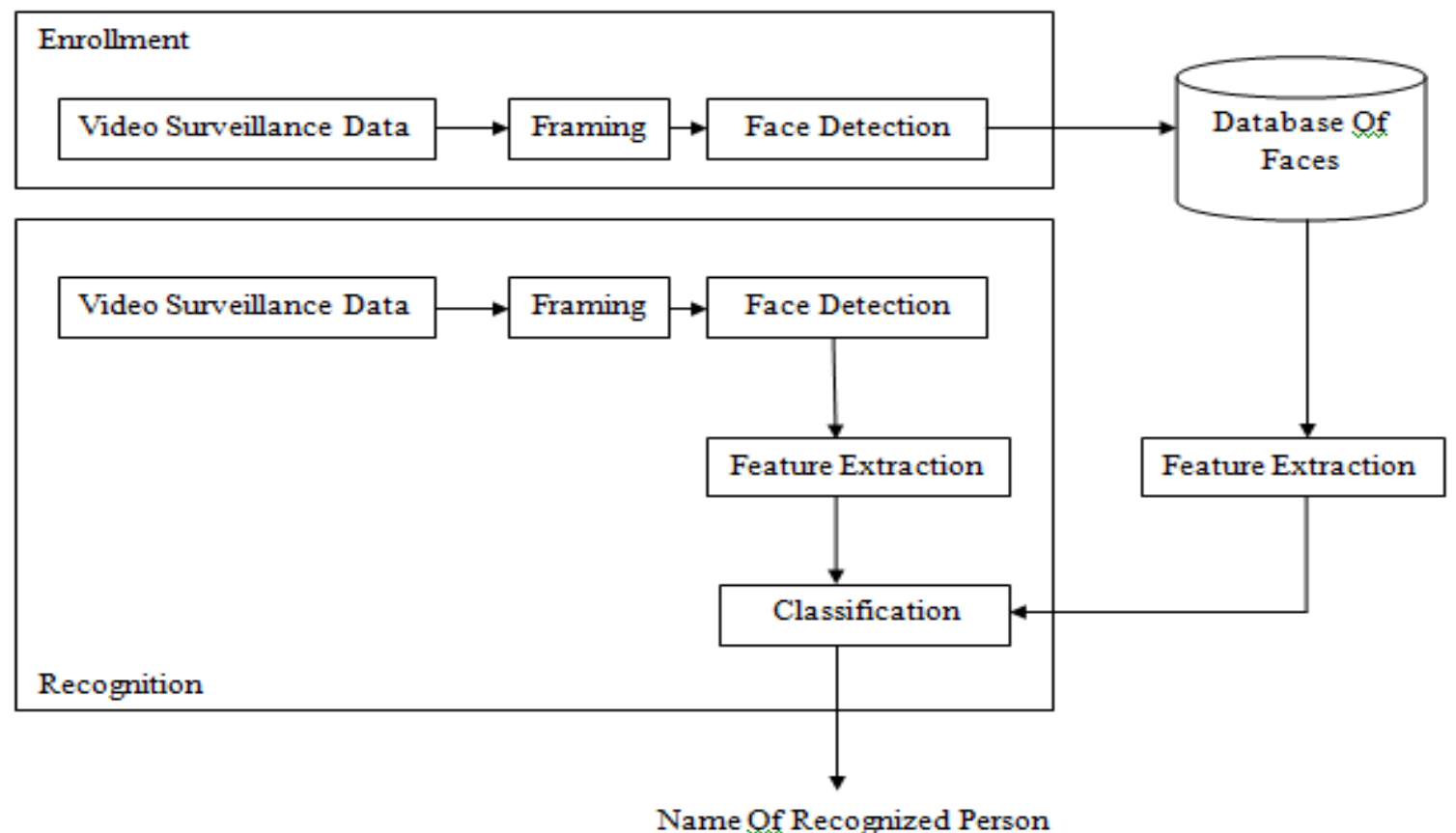

Fig. 1. Overview of the system

The pseudo code for proposed system is as follows.

ALGORITHM 1: Pseudo code of proposed System 1. Take a Video Surveillance Data from classroom

2. Apply viola Jones Algorithm [Face Detection]

3. Extract the detected faces

4. Perform pre-processing such as scaling, histogram equalization

5. Convert into grey scale Faces with different Orientation having dimension $100 * 100$

6. Store the images in database

7. Take video surveillance data for recognition

8. Apply LBPH classifier [Face Recognition]

9. Extract the features from detected faces

10. Compare Features

11. Put Student's Attendance based on recognized faces

Two main phases of the existing system are enrollment of students in the system and recognition of students and put attendance.

\section{A. Student Enrollment}

The student enrollment process involves the registration of student with the system. For registration process student need to come in front of the webcam of the laptop. At the time of registration, student's faces with different orientation are saved inside the database. In order to detect the student faces, we used Viola-Jones Face detection algorithm [2]. As compared with other face detection algorithms available [3] this method is much as faster. Viola-Jones face detection algorithm takes less computation time, but has high accuracy.

- Video Surveillance Data

We use surveillance video of classroom as a dataset

- Framing

The purpose of framing is to remove frames from videos that don't have a human as an object in it. After applying framing it helps to reduce the time required to detect the human face as the frames containing only human objects are available.

- Face Detection Algorithm

For face detection we are going to used vilo jones algorithm Viola Jones algorithm have many advantages over other face detection algorithm. We choose viola jones algorithm because it uses an integral image representation 
as well as AdaBoost due to this it will give better performance in different lighting conditions.

The step of viola jones algorithm is as follows:

Four steps are to be used for implementation of ViolaJones face detection algorithm are as follows:

a) Selection of Haar features

Haar features are digital image features used in face detection process. These features are moved over an image to detect an object. These features have two regions one is white and second is black. The computation carried out during haar feature selection is as follows eqn. 1.

$\mathrm{Val}=\Sigma$ (pixels in black region) $-\Sigma$ (pixels in white region)

In order to detect human faces the below features are applied on human faces and then threshold value is calculated by using above mentioned formula.
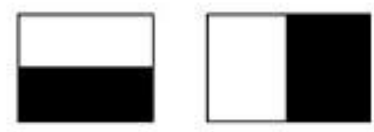

(a) Edge Features
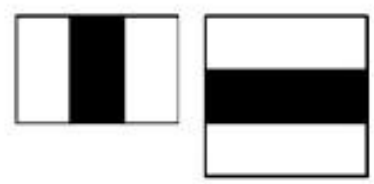

(b) Line Features

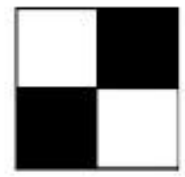

\section{(c) Four-rectangle features}

Fig.2 Types of Haar Features

Various types of haar features are used on an image is shown in the Fig.2.These features are developed by considering human face characteristics so that it will help us to detect the human face with great accuracy.

The features are selected based some of the properties associated with human faces. Such as region of the eyes has been often darker than region of the nose and cheeks. The second property is that eyes are darker than the bridge of the nose. But the same windows applying on the cheeks or any other place are irrelevant. So, among the 160000 features available appropriate features are selected using AdaBoost. In AdaBoost each feature is applied on all the training images. After that it finds the threshold value for each feature based on that faces are classified either as positive or negative. At the time of feature selection there will be errors or misclassification but we select the features with the minimum error rate.

b) Integral image representation

If the image is of dimension $24 * 24$ then that have near about 160000 features available to identify a human face. If we perform computation using all these features on the image then it will take too much time for computation. So to have a high-speed image is represented as an integral image. The integral image at location $(x, y)$, is the sum of the pixels above and to the left of $(\mathrm{x}, \mathrm{y})$, inclusive.

$$
\begin{array}{r}
\mathrm{ii}(\mathrm{x}, \mathrm{y})=\Sigma \mathrm{i}\left(\mathrm{x}^{\prime}, \mathrm{y}^{\prime}\right) \\
\mathrm{x}^{\prime} \leq \mathrm{x}, \mathrm{y}^{\prime} \leq \mathrm{y}
\end{array}
$$

Eqn.2 combines neighbor pixel values for easy of calculation.

Here ii $(x, y)$ is integral image and $i(x, y)$ is the original image.
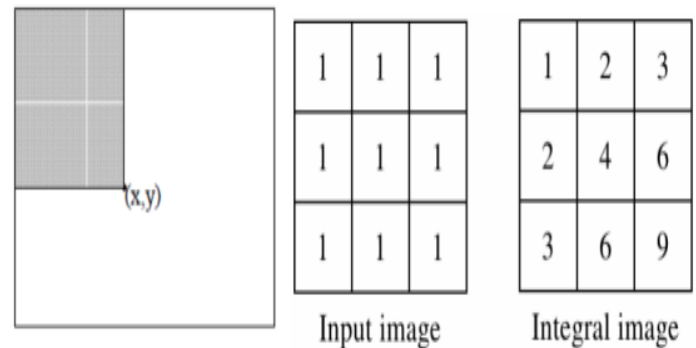

Fig.3 Integral image representation

\section{c) AdaBoost Training}

Though we have 160000 haar features but out of that only some features are capable to work independently .To overcome above limitation weak classifiers is grouped together. The process of grouping weak classifiers is called AdaBoost . The set of weak classifiers represented using below equation.

$$
\operatorname{hj}(x) \in\{+1,-1\}
$$

If we iteratively combine these classifiers the equation in the linear form is

$$
\mathrm{C}(\mathrm{z})=\theta(\Sigma \mathrm{ht}(\mathrm{x})+\mathrm{b})
$$

Eqn. 3 is use to declare classifier either as a weak or strong based on the value obtained from Eqn.1. Eqn. 4 combines the features having value greater than threshold value. It helps in the process of detection and recognition process.

The whole process carried out during AdaBoost training is shown in Fig. 4

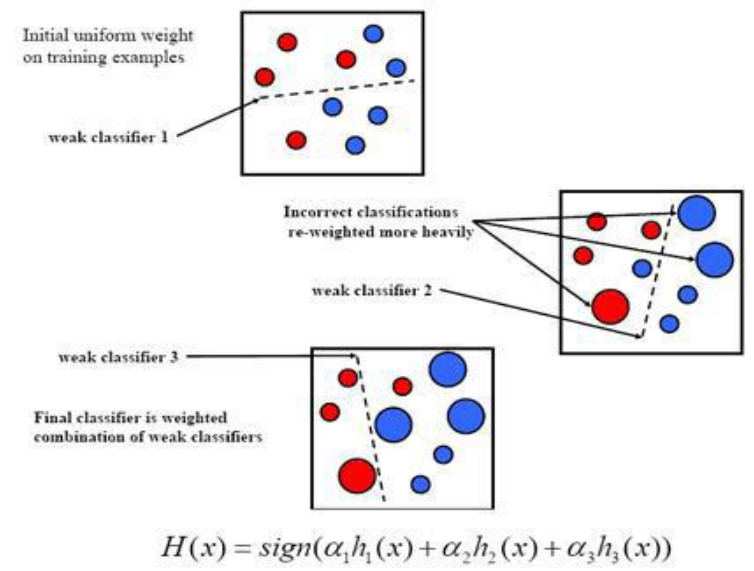

Fig.4 AdaBoost classifier grouping process 
d) Cascading classifier

As image having dimension 24*24 has near about 160000 features, If we use all these features to detect an object as a face in the image, then computational time will be more. so the alternative is to use cascading classifier in which at each stage classifiers are grouped together to detect the face. Image face

property not found then we simply discard that classifier and proceed to next classifier. Due to this computational time is reduced. The overview of the cascading classifier is shown in fig. 5

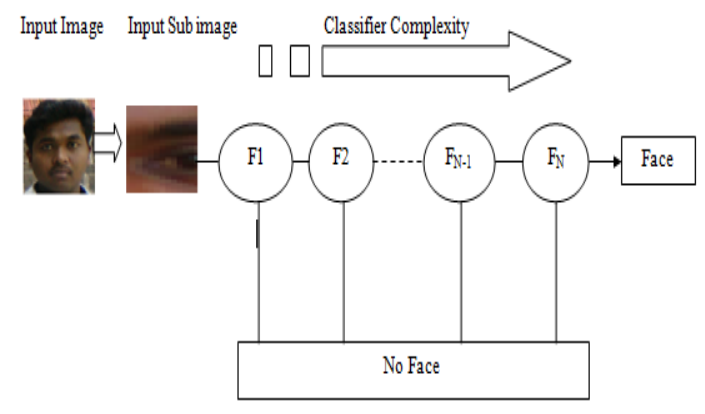

Fig.5 Cascading Classifier

- Database Database of Faces

We store several photos of a human faces with different orientation inside the database. Along with the faces, details such as name and roll number is also saved. This database is used at the time of recognizing the human face. The accuracy of this algorithm depends on the way we trained the system.

\section{B. Student Recognition}

Student recognition is the process of identifying students from the database. To recognize the human face we extract the features from the faces that are available in the frames and compared that features with the features of faces present in a database.

- Video Surveillance Data

There are two ways to use the video surveillance data, we either use live video from surveillance camera for recognition or we may use offline video which is already recorded and available somewhere in a system. Framing, Face Detection, and feature extraction are similar to that of student enrollment process.

For recognizing a human face we used PCA [6] [7] and LBP classifier the detailed operation of these algorithms is as follows.

The face recognition process carried out using LBP is as follows Thresholding is performed on input image such as if intensity value of the value of neighboring pixel is less then it is set to 0 else to 1 after that decimal value is formed by combing all neighboring values and put at center pixel. Image is subdivided into several parts and above operation is carried on each subpart and from each subpart local features are extracted. Based on obtained intensity values histogram of each part is calculated. All these histograms combined together to obtain resultant histogram for feature extraction.

\section{EXPERIMENTAL RESULTS}

A set of experiments carried out on surveillance data obtained from the mobile. The performance evaluation of the system is performed using this dataset.

The screenshots of various phases of attendance system are as follows:

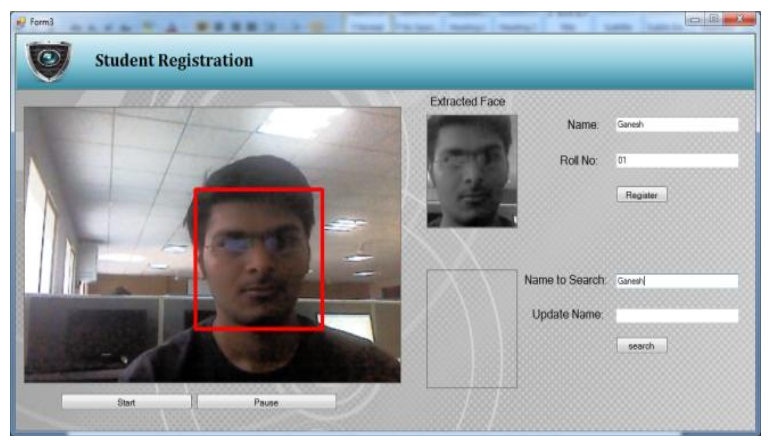

Fig.6 Student registration process

Fig. 6 is the registration process carried out in front of the webcam of the laptop. At the time of registration process, students faces with different orientation are saved inside the database.

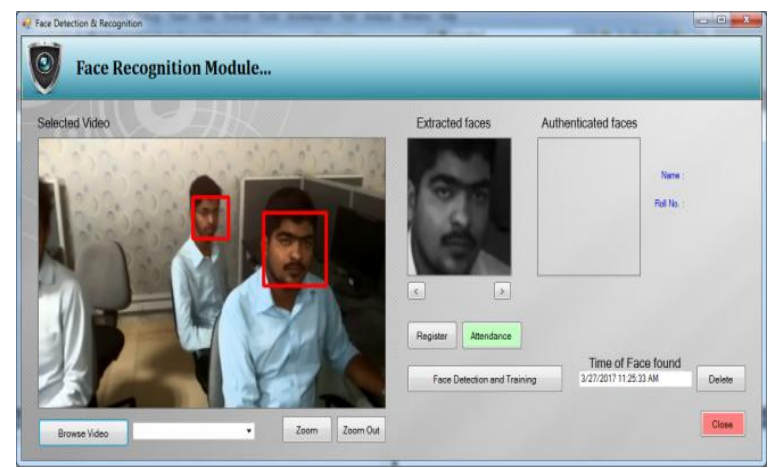

Fig.7 Video captured in practical lab

Fig.7 is the face recognition process with unregistered faces. Hence, even though face is detected not a single face mapped from database.

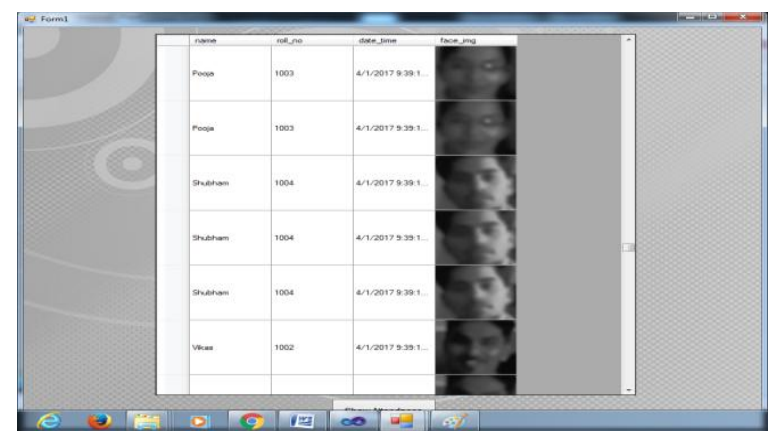

Fig.8 Faces present in database 


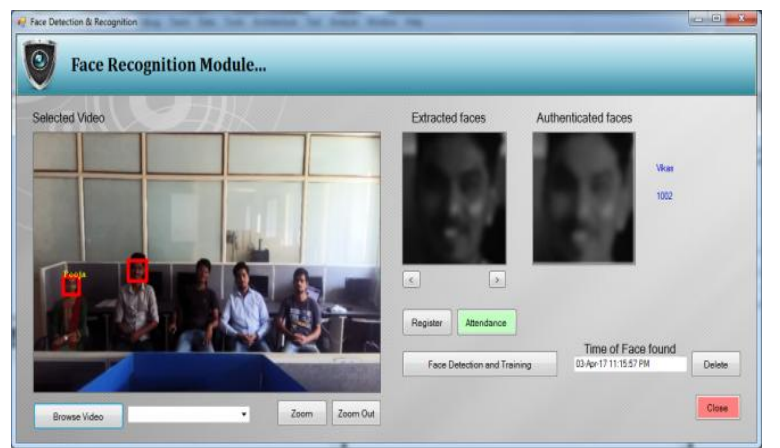

Fig. 9 shows the recognized faces at the time of attendance in a class.

Comparison of Holistic Face Recognition

\begin{tabular}{|c|c|c|}
\hline $\begin{array}{c}\text { SR. } \\
\text { NO }\end{array}$ & $\begin{array}{c}\text { Performance Evaluation } \\
\text { Conditions }\end{array}$ & $\begin{array}{c}\text { LBPH + } \\
\text { Threading }\end{array}$ \\
\hline 1 & False Face Detection & $20 \%$ \\
\hline 2 & Training Time & 13.8 Millisecond \\
\hline 3 & $\begin{array}{c}\text { Recognition Rate (Real } \\
\text { time Video) }\end{array}$ & $80 \%$ \\
\hline
\end{tabular}

- False Face Detection

False face detection rate is nothing but amount of false faces recognized incorrectly in given video time.

The formula to calculate false face detection rate is as follows

FFD $=$ False Face Detection

FRface $=$ False Recognized face

Tface $=$ Total faces

\section{FFD $=($ FRface $/$ Tface $) * 100$}

Table I: False face detection rate with varying number of students

\begin{tabular}{|c|c|}
\hline No of students & False face detection \% \\
\hline $0-10$ & $10 \%$ \\
\hline $20-40$ & $13 \%$ \\
\hline $40-70$ & $18 \%$ \\
\hline
\end{tabular}

The table shows that as the number of students inside the frame is increased then false face detection rate occurs.

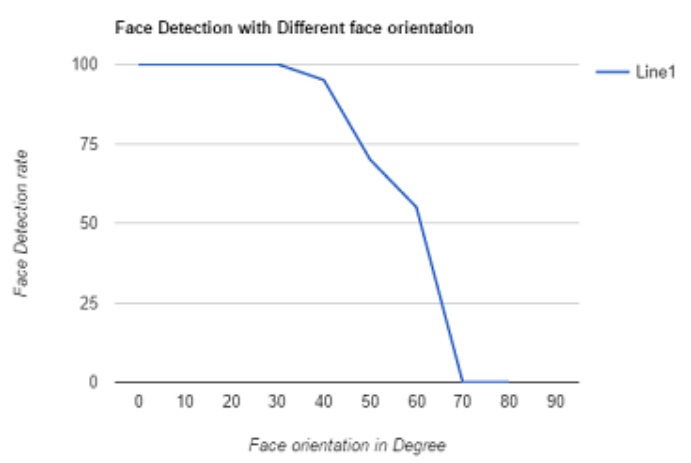

Fig.10 Face Detection rate vs. Face orientation
The Face Detection rate is $100 \%$ till the frame containing 40 students but above that face detection rate reduced slightly to $3 \%$.

Fig. 10 is graph plotted between face orientations in degree against face detection rate. It shows that as face orientation goes beyond $50^{\circ}$ face detection rate decreases continuously.

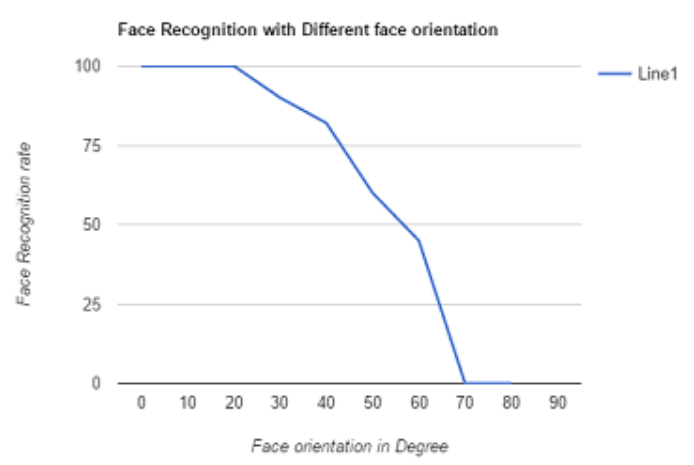

Fig.11 Face Recognition rate vs. Face orientation

Fig. 11 is graph plotted between face orientations in degree against face recognition rate. It shows that as face orientation goes beyond $35^{0}$ face recognition rate decreases continuously.

- Training Time

Training time required for image is nothing but time required to extract one human face.

The formula to calculate training time is as follows

Vtime $=$ video timing in sec.

Eface $=$ No. of extracted faces.

Ttime $=$ Avg. Ttime for extracted face in millisecond .

Ttime $=($ Eface $/$ Vtime $) * 1000$

- Recognition Rate (Real time Video):

Face recognition rate is nothing but amount of faces recognized correctly in given video time

RTime $=$ Recognition Time

RFace $=$ Recognized face

\section{RTime $=($ RFace $/$ Total Face $) * 100$}

\section{CONCLUSION}

The conclusion comes out of our system is that the already existing systems support attendance of students only when individual student will come in front of camera but our system allows group of student . 


\section{REFERENCES}

[1] Lei, Zhen, et al, “ Real-time face detection and recognition for video surveillance applications ", Computer Science and Information Engineering, 2009 WRI World Congress on. Vol. 5. IEEE, 2009.

[2] Viola, Paul, and Michael J. Jones, “ Robust real-time face detection ”,International journal of computer vision 57.2 (2004):137-154.

[3] Degtyarev, Nikolay, and Oleg Seredin, "Comparative testing of face detection algorithms", Image and Signal Processing. Springer Berlin Heidelberg 2010. 200-209.

[4] T. Lim, S. Sim, and M. Mansor," RFID based attendance system ", in Industrial Electronics and Applications, 2009. ISIEA 2009. IEEE Symposium on, vol. 2. IEEE, 2009, pp. 778782.

[5] S. Kadry and K. Smaili," A design and implementation of a wireless iris recognition attendance management system ", Information Technology and control, vol. 36, no. 3, pp. 323329, 2007

[6] Faruqe, M.O. and Hasan, M. A. M.(2009). " Face recognition using PCA and SVM. " Proceedings of the 3rd International Conference on AntiCounterfeiting, Security and Identification in Communication, Aug. 20-22, Hong Kong, pp: 97-101.

[7] Turk, M., Pentland, A.: “ Eigenfaces for recognition " . Journal of Cognitive Neuroscience 3 (1991) 71-86

[8] Ojala, T., Pietik"ainen, M., M“aenp"a“a, T.: “ Multiresolution grayscale and rotation invariant texture classification with local binary patterns." IEEE Transactions on Pattern Analysis and Machine Intelligence 24 (2002) 971-987

[9] Ojala, T., Pietik"ainen, M., Harwood, D.: “ A comparative study of texture measures with classification based on feature distributions. Pattern Recognition " 29 (1996) 51-59

[10] K.Senthamil Selvi, P.Chitrakala, A.Antony Jenitha ," Face Recognition Based Attendance Marking System" Ijcsmc, Vol. 3, Issue. 2, 2014

[11] K. Mohamed and C. Raghu, "Fingerprint attendance system for classroom needs," in India Conference (INDICON), 2012 Annual IEEE. IEEE, 2012, pp. 433-438.

[12] T. Lim, S. Sim, and M. Mansor, "Rfid based attendance system," in Industrial Electronics \& Applications, 2009. ISIEA 2009.IEEE Symposium on, vol. 2. IEEE, 2009, pp. 778-782.

[13] J.Chatrath, P.Gupta, P.Ahuja, A.Goel, "Real Time Human Face Detection and Tracking" International Conference on Signal Processing and Integrated Networks (SPIN) Volume 1, Issue 1,pp. 705-710, 2014.

[14] T. Ahonen, A. Hadid, and M. Pietik"ainen, "Face recognition with local binary patterns," in Computer Vision-ECCV 2004. Springer, 2004, pp. 469-481

[15] T. A. P. K. K. L. P. M. L. M. P. A. W. G. D. P. J. G.. RoshanTharanga, S. M. S. C. Samarakoon, "Smart attendance using real time face recognition," 2013. 